were largely dilated and unalterable; and I do not think he could see anything held close to him. He could not speak distinctly; and deglutition was much impaired.

In the night, he was sick twice, and the bowels were relieved once. He had no rest at all.

In the morning, at 8, I saw him again. He could then speak, and knew what was said to him. His face was not flushed. I gave him some calomel and jalap, which acted freely; and with each motion there was marked amelioration of his symptoms. At 1 p.M., when I saw him, he was well, with the exception of some irregularity in the movement of his legs.

At my second visit (five hours after the commencement of the symptoms), I was struck by the resemblance of the case to the one reported above, and made careful inquiries as to what he had eaten, and whether he had access to deadly nightshade or any of the poisonous solanaceæ; but could obtain no clue. But, on the second day, he confessed to having drunk something out of a bottle, which I found to contain belladonna lotion; and, by a rough calculation, he must have had about twenty grains of the extract.

REMARKs. In both these cases, the treatment was all but $n i l$; and so they seem to confirm the opinion expressed by Dr. Skinner at Liverpool (as reported in the Journal of November 18th, 1865), that Nature acts well in such cases. They also point to the stimulant, rather than sedative, action of belladonna.

\section{INVERSION OF THE UTERUS.}

By John Birchenall, Esq., Macclesfield.

Dr. Marion Sims' cases of inversion of the uterus, recently reported in the JourNaL, reminded me that a similar misadventure occurred in my own practice about thirty-five years ago.

My patient was a single woman, about 25 years of age, rather tall and spare, of substrumous diathesis, fair complexion, and nervous temperament. It was her first pregnancy. The pains of labour came on early in the morning; and at eight o'clock A.r., I found the head of the child occupying the pelvic cavity; the os uteri thin and considerably dilated; and the pains, which were brisk, recurring every two or three minutes, until 11 o'clock, when delivery was accomplished in the natural way. After the lapse of some ten or twelve minutes more, as the uterus had contracted, and the placenta was unexpelled, I made gentle traction by the cord (which I had been accustomed to do when there was nothing to contraindicate it) for the purpose of exciting the expulsive efforts. Though no force was employed, this was immediately followed by a shriek on the part of my patient, and the simultaneous protrusion into the vaginal orifice of the undetached placental mass, overlapped on its lower margin by the smooth and livid edge of the inverted uterus. In the dilemma, I sent for an experienced accoucheur; but, as he was not in the way, I proceeded carefully to detach the placenta. This was easily effected, as there was no adhesion. I then replaced the uterus within the pelvis, and, grasping the fundus within the ends of my fingers and thumb, gave a moulding pressure to the organ, which, to my great gratification, after a few seconds, receded by a sudden involution, and everything returned to its normal condition. There was no hæmorrhage, nor any subsequent inconvenience, to retard tine recovery.

My patient continued in perfect health, nursing her child during the few months she remained in
Macclesfield; but, as she left the town before her infant was weaned, I had no opportunity of ascertaining the subsequent functional condition of the uterus.

\section{Cramsactions of apanches.}

\author{
BATH AND BRISTOL BRANCH.
}

ON THE TREATMENT OF RETENTION OF THE MENSES FROM OCCLUSION OF THE VAGINA AND UTERUS.

By W. Michell Clarke, Esq., Clifton.

$$
\text { [Read Octover 25th, 18065.] }
$$

There was admitted into the Bristol General Hospital, under the care of Dr. Martyn, on December 31st, 1864, a young woman, who was suffering from retention of urine; her age was 28 . Upon attempting to pass a catheter, the house-surgeon found that the vagina was completely occluded, and the patient was transferred to me.

She gave the following history. Eight years previously she was delivered of a still-born child, which was, at the time of its birth, in a state of putrescence. She was a long time recovering, was confined to her bed several months; and, when at length she was able to get up, she discovered that her vagina was perfectly closed. She had not menstruated since the date of the confinement; but at her catamenial periods, she had been accustomed to have a good deal of bearing down pain, and a sharp stitch in her side, which were usually relieved by diarrhœa. In the intervals, her health was good, and she never experienced any vicarious discharge of blood. For the last four months, however, she had suffered from gradually increasing difficulty of micturition until on December $9 \mathrm{th}$, she found herself unable to pass urine at all, and since that date she had had the catheter passed several times, the introduction of the instrument having been, I believe, attended with considerable difficulty. I found that the orifice of the urethra was much dilated, so that the difficulty arose from no narrowness of the canal; but the catheter required to be depressed very much at its outer extremity, in order to its introduction. Why the urethra was so much dilated, I cannot tell, as my patient was a single woman, and not, I believe, in the habit of indulging in sexual intercourse. In similar cases that have been recorded, of complete occlusion of the vagina, there was no doubt that the urethra had taken the place of the vagina in coition.

After emptying the bladder, I found a large tumour in the abdominal cavity, corresponding in size and shape to the pregnant uterus of the fifth or sixth month; and there was, moreover, a smaller tumour projecting from the right side of the larger, which felt like a fibrous growth. The principal tumour gave no sensation of fluctuation, but communicated a soft doughy feeling to the hand.

The vagina was completely closed at its orifice by an extensive cicatrix, which drew the urethra and rectum together, and it seemed not easy to say to what depth this cicatrix extended; but after a very careful examination, I could not discover the smallest perforation; there were two very small openings, but they only admitted a probe a very little way.

On introducing the finger into the rectum, a large tumour was felt pressing back upon that viscus, and occuping the most of the pelvic cavity; and this was further found to correspond with the swelling felt through the abdominal wall. I could have no doubt 